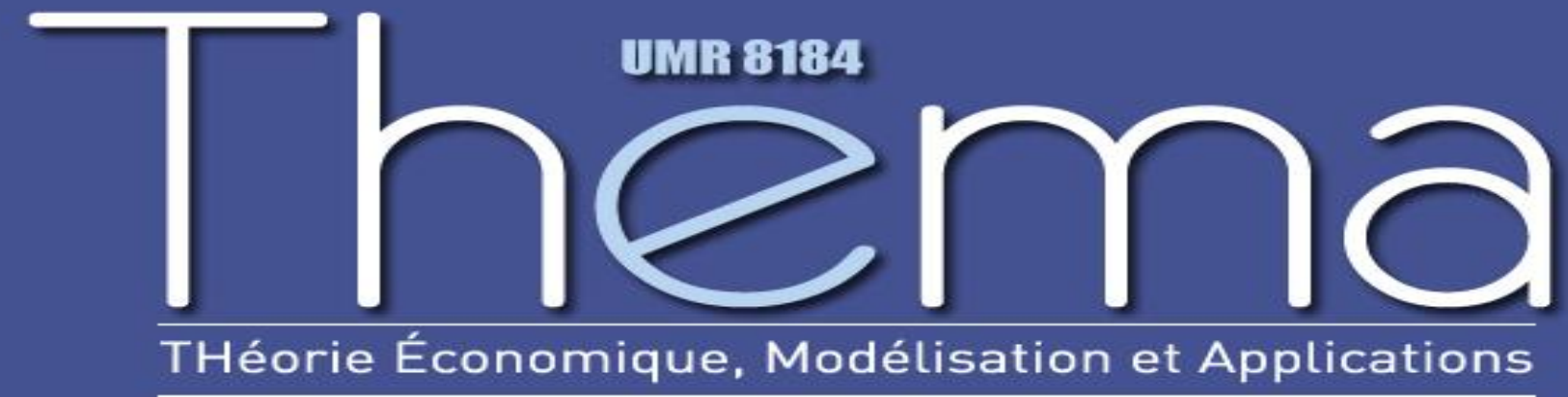

Thema Working Paper $\mathrm{n}^{\circ}$ 2012-43

Université de Cergy Pontoise, France

Welfare Comparisons, Economies of Scale and Equivalence Scale in Time Use

Hélène Couprie Gaëlle Ferrant

December, 2012 


\title{
Welfare Comparisons, Economies of Scale and Equivalence Scale in Time Use *
}

\author{
Hélène Couprie ${ }^{\dagger}$ and Gaëlle Ferrant ${ }^{\ddagger}$
}

8th December 2012

\begin{abstract}
How does within couples' time-use interactions generate welfare in the family? In this paper we model economies of scale in time use. Following Browning, Chiappori and Lewbel (2006), we allow intrahousehold bargaining power to affect the distribution of welfare gains in the family. We estimate the model using the UK Time Use Survey (2000). Results suggest that two single individuals living apart need about $2 \mathrm{~h} 15$ more to achieve the same utility level as when living in a couple. A woman requires on average $55 \%$ of the couple time-resources to be as well-off as when living alone. Time-poverty line is on average $11 \mathrm{~h}$ per individual a day.
\end{abstract}

\footnotetext{
${ }^{*}$ We thank the participants at SMYE 2012, Bernard Fortin, Arthur Lewbel, Olivier Bargain, Olivier Donni, Frederic Vermeulen, Bram de Rock, Laurens Cherchye, Catherine Sofer and François Gardes for their comments.

†Université de Cergy-Pontoise, THEMA, Les Chênes, 33 Boulevard du Port, 95011 Cergy-Pontoise Cedex, France (email:helene.couprie@u-cergy.fr ).

${ }^{\ddagger}$ Centre d’Economie de la Sorbonne-Université Paris I Panthéon Sorbonne, 106-112 Bd de l'Hôpital, 75647 Paris Cedex 13, France (email:gaelle.ferrant@univ-paris1.fr).
} 


\section{Introduction}

Equivalence scales are widely used to make interpersonal welfare comparisons of households of different sizes, compositions or characteristics. They allow converting observed expenditures of different types of households in comparable units (Lechene, 1993; Banks and Lewbel, 1997). For example, sharing the accommodation, heating and so forth generates economies of scale and welfare gains that could be measured using equivalence scales. These gains vary depending on household composition and income. They are widely applied to define poverty lines for example. In practice, welfare level is exclusively defined on a good consumption basis (Nelson, 1993). However, the disposition of time can be viewed as "the ultimate source of utility" (Zeckhauser, 1973), and time-poverty is a concept that is of growing interest in the literature (Vicker, 1977; Goodin et al., 2005; Hamermesh and Jungmin, 2007).

In this paper, we estimate a household's time allocation model that allows identifying economies of scale in the time allocation of the family. We define equivalence scales based on time-use. This paper brings a better understanding of how family interaction converts time into welfare. It allows answering the following questions: "How much time does a couple save by living together versus living apart?" "How much time would a single female require to attain the same utility that she would have if she lived in a couple?" Indeed, living in couple means saving time, because, for example, cooking for two does not require twice more time than cooking for one. Thus, living together and sharing create economies of scale within household, meaning that a widow needs more free-time than what she has to achieve the same welfare she had once living in a couple. To our knowledge, except Van Hoa and Ironmonger (1989), there is no such attempt in the economic literature. 
From a methodological viewpoint, our paper is situated in the literature that solves theoretical controversies regarding equivalence scales estimation. Traditional equivalence scales define an equivalent income at the household level, answering the following question: "what is the expenditure level required by a single household to be as well off as if it was a household with several members?" . To this aim, utility cardinalization is required (see Pollak and Wales, (1979, 1995); Gronau, 1988; Nelson, 1988; Browning et al., 2006 for more details). Second, traditional definition assumes that within households, welfare levels are equalized (Nelson, 1993). Recently, Browning et al. (2006) (BCL hereafter) stated that in the standard approach "the notion of a household utility is flawed. Individuals have utility, not households. What is relevant is not the 'preferences' of a given household, but rather the preferences of the individuals that compose it.". They proposed a new definition of equivalence scale that maintains ordinality of utilities and hence is free from such drawback. BCL estimate adult-indifference scales. To this aim they defined individual equivalent incomes that answer the following question: "what is the expenditure level required by a single individual to be as well off as if he was member of a household with several members?". In this case, instead of comparing cost functions of households of different types, an unique individual cost function in two different situations is compared, allowing analyzing the possible intra-household variation in the standard of living.

BCL analysis is based on a collective representation of household decisionmaking process. The household is the scene of bargaining process among its members, where the sharing rule characterizes the bargaining power of each member (Sofer, 1999, Browning et al., 2006; Apps and Rees, 1988; Browning and Chiappori, 1998; Vermeulen, 2002; Chiappori and Donni, 2006; Fortin 
and Lacroix, 1997). In this case, household utility function is defined as a weighted sum of family members individual sub-utility functions. Household behavior is also Pareto-efficient. To capture economies of scale in consumption, BCL introduce a consumption technology function that characterizes the intra-household publicness of goods consumption or positive externalities associated with the consumption of goods within the household.

Vermeulen and Watteyne (2006) and Lewbel and Pendakur (2008) provide an alternative specification that eases identification. Vermeulen and Watteyne (2006) propose to give up the consumption technology function. They define a priori goods that are privately consumed and those which are publicly consumed (accommodation and heating, for example). Bargain and Donni (2012) and also Lewbel and Pendakur (2008) provide a model relying on Engel curves estimates, without price variation. Thus, Bargain and Donni (2012) defined a new concept of child cost using this framework. Cherchye, De Rock and Vermeulen (2012) use the BCL framework to analyze economic well-being and poverty among elderly.

Our model brings new elements to this framework. We introduce a timeuse technology function that reflects intra-household economies of scales in time. The links between good and time consumption are clarified thanks to a time separability assumption which we test in the empirical part. Our contribution brings a first stone in the building of a complete model of intrahousehold welfare interaction due to consumption and leisure. We apply the model to UK time-use data. Results show that two singles living apart need 2h15 hours more free time to achieve the same utility level as living in couple. A woman requires on average $55 \%$ of joint time-resources to be as well-off as when she lives alone and men 52\%. Time-poverty lines are also defined.

The theoretical model is presented in a first part, then the empirical ana- 
lysis and results are described in a second part. The last section concludes.

\section{Household's Daily Time allocation Choice}

Time-use allocation decision is analyzed on a short term daily basis, i.e. the allocation of spare time amongst 'pure' leisure, housework, sleeping, personal care, and commuting. Medium term choices, such as consumption or hours of work are taken as given. We focus on dual-earner full-time working couples or single individuals without children. Household members', identified by subscript $i=f, m$, are endowed with a well-behaved utility function depending on a vector of $\mathrm{K}$ commodities (denoted $\mathbf{z}): U_{i}\left(\mathbf{z}_{i}\right)$.

The predetermination of consumption and hours of work choice makes sense. Indeed, the usual worker receives his wage and salary every month. The labor contract defining wages and working hours is often signed for a longer time period. Working hours can evolve for shorter time periods, but this might mostly come from the employer's side to insure higher flexibility of the production process. In this perspective, hours of work could be modeled as time constraints.

We present the model under a separability assumption which requires that hours of work and consumption do not impact daily time allocation, except through an income effect. If separability does not hold then Pareto-efficiency in conditional program is not sufficient to guarantee Pareto-efficiency for the unconditional one (Blundell, Chiappori and Meghir, 2005). In this case identification still holds but conditionally on medium run choices. The reach of the analysis is restricted. Separability between consumption and time-uses will be tested in the empirical part. 


\subsection{Time-use Technology Function}

The time-use technology function is analogous to the consumption technology function (see Browning et al., 2006 and Cherchye, De Rock and Vermeulen, 2012). The commodity vector $\mathbf{z}$ is assumed to be linearly produced within the household using its members' uses of time: $\mathbf{z}=F\left(\mathbf{t}_{f}, \mathbf{t}_{m}\right)$. Here, the time-use technology function takes the form of a linear Barten-type technology: $\mathbf{z}_{f}+\mathbf{z}_{m}=\mathbf{z}=\mathbf{A}\left(\mathbf{t}_{f}, \mathbf{t}_{m}\right)$. In single households, the different time-use activities are directly considered as commodities entering in the individual utility function. This implies that $\mathbf{z}_{i}=\mathbf{t}_{i}$.

The interpretation of the relationship is twofold. On one hand, it can simply be viewed as a household production process with constant returns to scale and perfect substitutability of time-use activities within the household and across activities. In this view, commodities produced by the household are assignable to household members. In this specification, the time-use technological process can be interpreted as a process describing how an aggregate private good commodity vector $\mathbf{z}=\mathbf{z}_{f}+\mathbf{z}_{m}$ is produced at the household level and then shared amongst household's members. Production of a diner could easily enter in this category, while other uses of time could be hardly perceived through this production perspective.

On the other hand, it can be viewed as a time-use generalization of the consumption technology function. The consumption technology function proposed by BCL transforms household effective purchases into private good equivalent bundles $\left(\mathbf{z}_{i}\right)$. The time-use technology function that is proposed in this paper represents what could be the economies of scales in terms of time in the household. It transforms time effectively spent $\mathbf{t}_{i}$ into private time equivalent $\mathbf{z}_{i}$. More precisely, it allows defining equivalence scale on an individual basis (denoted adult indifference scales). Knowing this, this tech- 
nology will help to understand how family status (being single or in couple) impacts individual welfare. In other words, does living in a household comparing to living alone allows individuals to save time? Sharing and jointness of time-use within the family probably induces welfare gains. The time-use technology function incorporates welfare externalities linked with the use of time. Jointness of household production or even leisure activities may induce welfare gains that could be reinterpreted in terms of economies of time ${ }^{1}$

Contrary to BCL, our model adopts a non-additive technology function. Nevertheless, due to assignability of time use, generically nonparametric identification results hold $!^{2}$ Therefore, the shadow prices are different for male and female. Intuition suggests that the first interpretation corresponds best to the time devoted to domestic chores and the second one corresponds best to leisure activities.

We now turn to the interpretation of the $\mathbf{A}$ matrix. The matrix $\mathbf{A}$ takes the shape of a bloc-diagonal matrix. On the diagonal of the matrix, we have the direct effect of own time on the commodity consumption, which could be normalized to 1 . The other elements $\boldsymbol{\alpha}$ of the matrix $\mathbf{A}$ associated with purely private time are equal to 0 those associated with purely public time are equal to 1 . Elements comprised between 0 and 1 describes time having a public and a private component. We call $\boldsymbol{\alpha}$ the degree of publicness of the time where $0 \leq \boldsymbol{\alpha} \leq 1$. The link between the private equivalent $z_{f}^{k}$ and $z_{m}^{k}$ for time-activity $k$ and the household members' time-uses $t_{f}^{k}$ and $t_{m}^{k}$ are

\footnotetext{
${ }^{1}$ The interpretation in terms of economies of scale is more delicate since private equivalent goods are directly recovered on an individual basis from this technology function. Hence, disentangling production and consumption argument in the understanding of this consumption technology is less clear than in BCL model. On the other hand, the assignability property of time-use activities has the advantage of facilitating identification of the model.

${ }^{2}$ It is worth noticing that BCL show that the model is generically non-parametrically identified meaning that identification will only fail if the utility function or the technology function are too simple.
} 
defined in the following way:

$$
\left\{\begin{array}{l}
z_{f}^{k}=t_{f}^{k}+\alpha^{k} t_{m}^{k} \\
z_{m}^{k}=t_{m}^{k}+\alpha^{k} t_{f}^{k}
\end{array}, \text { for } k=1, \ldots, K\right.
$$

\subsection{Time-use Demand for Singles}

We first describe the behavior of single households. For sake of simplicity the household's subscript is omitted. In the medium run, the household chooses his consumption $C$ and a total time of leisure $\tilde{T}$ (or equivalently hours of work $H$ since $\widetilde{T}=T-H)$. On a daily basis, each rational agent maximizes a well-behaved short-run utility function, denoted $U$, with respect to a daily time-use allocation, subject to a time-budget constraint, as well as a time constraint. We denote $\mathbf{t}=\left(t^{1}, \ldots, t^{K}\right)$ the individual time-use vector and $\widetilde{T}$, the daily spare time:

$$
\left(P^{s}\right)\left\{\begin{array}{l}
\max _{t_{i}} U_{i}\left(t_{i}\right) \\
\text { st. } \Sigma_{k=1}^{K} t_{i}^{k}=\widetilde{T}_{i} \\
\text { st. } t_{i}^{k} \geq 0, t_{i}^{k} \leq T, H_{i}>0, H_{i} \leq T
\end{array}\right.
$$

Daily time-use demands can be equivalently written in the following way:

$$
t_{i}^{k}=t_{i}^{k}\left(\widetilde{T}_{i}\right) \text { for } k=1, \ldots, K
$$

In the single individual case, prices of different activities are the same. This hinders identification of price elasticities. However, since wage rates, as well as the short run spare time $\widetilde{T}$ varies across individuals, under some parametric restrictions described later in the text, it is possible to identify these elasticities. 


\subsection{Commodities and Time-use Demand for Couples}

Couple's daily allocation of time is supposed to follow a collective model (Apps and Rees, 1988; Browning et al., 1994). Time allocation in the short run (i.e. on a daily basis) is efficient and taken conditional on medium run consumption and labor supply choices. For the couple, individuals timeconstraints are $\Sigma_{k=1}^{K} t_{f}^{k}=\widetilde{T}_{f}$ and $\Sigma_{k=1}^{K} t_{m}^{k}=\widetilde{T}_{m}$. Therefore, household daily time-allocation program $\left(P^{c}\right)$ is for the case of couples:

$$
\left(P^{c}\right)\left\{\begin{array}{l}
\max _{t_{f}, t_{m}} \mu \cdot u_{f}\left(z_{f}\right)+u_{m}\left(z_{m}\right) \\
\text { st. } \Sigma_{k=1}^{K} t_{i}^{k}=\widetilde{T}_{i} \\
\text { st. } Z_{f}^{k}=t_{f}^{k}+\alpha^{k} t_{m}^{k} \\
\text { and } Z_{m}^{k}=t_{m}^{k}+\alpha^{k} t_{f}^{k} \\
\text { st. } t_{i}^{k} \geq 0, t_{i}^{k} \leq T
\end{array}\right.
$$

The Pareto weight $\mu$ generally depends on prices, incomes and eventually distribution factor ${ }^{3}$ It reflects the weight of individual sub-utility in the household decision-making process. So generally, if the commodities are normal goods, higher is the weight for one household member, greater is his share of household private goods.

We now focus on interior solutions ${ }^{4}$. To this aim, broad definitions of type activities will be chosen in order to make sure that all household mem-

\footnotetext{
${ }^{3} \mathrm{~A}$ distribution factor is a variable which affects bargaining power but not preferences of individual household members or the joint budget set.

${ }^{4}$ Extending the empirical approach to corner solutions would allow considering a greater number of time-use activities. However, this extension in a structural daily time-use allocation case is not straightforward. In structural collective models, considering nonparticipation of labor supply choices is now relatively standard (Donni, 2009; Bloemen, 2010), it is not the case when considering several uses of time. Furthermore, in the case of daily time-use, additional identification assumptions would be necessary to disentangle infrequent answers from actual non-participation choice in some daily activities (Browning and Bonke, 2006). Given the novelty of the approach undertaken here, we keep this problem for further research.
} 
bers do spend a positive amount of their time in each of them. Following BCL, solutions of program $\left(P^{c}\right)$ can be rewritten as solution of individual decentralized program $\left(P^{d}\right)$ using Lindahl (1919) prices, denoted $\boldsymbol{\pi}$, as well as a share of household second full income denoted by $\eta_{i}$ :

$$
\left(P^{d}\right)\left\{\begin{array}{l}
\max u_{i}\left(\mathbf{z}_{i}\right) \\
\text { st. } \Sigma_{k=1}^{K} \pi_{i}^{k} z_{i}^{k}=\eta_{i}
\end{array}\right.
$$

In the decentralized case, the sharing rule $\eta$ is the share of income an individual living in a couple can spend on private commidities. The cost of private commodities is evaluated at a shadow price, denoted $\pi$, that itself depends on how economies of scales in time are generated in the household. Because of separability assumption between medium and short run, the Pareto weight and the associated sharing rule in the conditional program only depends on household time budget (which is the equivalent of second step income in a two-step budgeting procedure) as well as distribution factors. In particular, prices, such as wage rates do not appear. If separability does not hold, then efficiency at the sub-level cannot be guaranteed and prices could impact preferences for daily commodities $\mathbf{z}$, as well as the sharing rule. Pareto weights and sharing rule refer both to the bargaining power in the decision process: higher is the weight $\mu$ and the sharing rule $\eta_{f}$, greater are the private time equivalent consummed by the female individual $z_{f}^{k}$. Nevertheless, in principle, the identification of the sharing rule $\eta$ is preferred, as that does not depend upon any cardinalizations of the utility functions $U_{f}$ and $U_{m}$, contrary to the Pareto weight $\mu$.

Each time-use activity has an implicit shadow value that can be computed (see Appendix A). Individual shadow prices do vary within the household, 
meaning that individuals have different marginal valuation for the activities:

$$
\left\{\begin{aligned}
\pi_{f}^{k} & =\frac{w_{f}\left(\widetilde{T}_{m} \lambda_{f}-\widetilde{T}_{f} \lambda_{m} \alpha^{k}\right)}{\widetilde{T}_{m} \lambda_{f}\left[1-\left(\alpha^{k}\right)^{2}\right]} \\
\pi_{m}^{k} & =\frac{w_{m}\left(\widetilde{T}_{f} \lambda_{m}-\widetilde{T}_{m} \lambda_{f} \alpha^{k}\right)}{\widetilde{T}_{f} \lambda_{m}\left[1-\left(\alpha^{k}\right)^{2}\right]}
\end{aligned}\right.
$$

where $\lambda_{i}$ is the Lagrangian multiplier associated with the individual timeconstraint which represents the opportunity cost of domestic time for the household member $i$.

From program $\left(P^{d}\right)$ conditional demands for $\mathbf{z}_{i}$ might be obtained in a way similar to what we obtained for singles (Equation (3)), except that in this case, prices related to each time-use activity are shadow prices that might differ from one activity to another and by individuals. The time budget depends on the share of household full income obtained by the individual, $\eta_{i}$ :

$$
z_{i}^{k}=h_{i}^{k}\left(\pi_{i}^{1}, \ldots, \pi_{i}^{K}, \eta_{i}\right)
$$

Plugging preceding expression into Equation (1) leads to structural time-use demands:

$$
\left\{\begin{array}{l}
t_{f}^{k}=\frac{1}{1-\left(\alpha^{k}\right)^{2}} h_{f}^{k}\left(\pi_{f}^{1}, \pi_{f}^{2}, . ., \pi_{f}^{K}, \eta_{f}\right)-\frac{\alpha^{k}}{1-\left(\alpha^{k}\right)^{2}} h_{m}^{k}\left(\pi_{m}^{1}, \pi_{m}^{2}, . ., \pi_{m}^{K}, \eta_{f}\right) \\
t_{m}^{k}=\frac{1}{1-\left(\alpha^{k}\right)^{2}} h_{m}^{k}\left(\pi_{m}^{1}, \pi_{m}^{2}, . ., \pi_{m}^{K}, \eta_{m}\right)-\frac{\alpha^{k}}{1-\left(\alpha^{k}\right)^{2}} h_{f}^{k}\left(\pi_{f}^{1}, \pi_{f}^{2}, . ., \pi_{f}^{K}, \eta_{m}\right)
\end{array}\right.
$$

\section{Identification}

The reduced form time-use demands of couple depends only on observables $\widetilde{T}_{i}$ as well as observed heterogeneity variables $\mathbf{x}$ :

$$
\left\{\begin{aligned}
t_{f}^{k} & =t_{f}^{k}\left(\widetilde{T}_{f}, \widetilde{T}_{m}, \mathbf{x}\right) \\
t_{m}^{k} & =t_{m}^{k}\left(\widetilde{T}_{f}, \widetilde{T}_{m}, \mathbf{x}\right)
\end{aligned}\right.
$$


Structural time-use demands are defined above in Equation (6). They depend on commodity demands of both couple members, $h_{f}$ and $h_{m}$, and on parameters of the time-use technology function, A. Commodity demands depend themselves of shadow prices $\boldsymbol{\pi}_{f}$ and $\boldsymbol{\pi}_{m}$ that were derived in the preceding section. These prices are themselves a known function of observables as well as parameters of interest $\boldsymbol{\alpha}$ and Lagrangian multipliers $\lambda_{f}, \lambda_{m}$ which represents the opportunity cost of domestic time.

The identification question is the following. Can we identify the $K$ parameters of the time-use structural technology function and the two opportunity costs $\lambda_{f}, \lambda_{m}$ from the observation of the reduced form time-use functions (Equation (7D) obtained for couples and singles?

To this aim, some conditions must be satisfied. First, we are going to assume, as is usual in this literature, that conditionnal preferences for time do not vary from one family status to another. Second, time-use demands, and especially price elasticities should be identified from the estimates obtained on singles.

Assumption 1: Conditional on characteristics, commodity demands $h_{f}$ and $h_{m}$ are the same whatever the marital status.

This assumption, despite controversial, is common in the literature (Couprie, 2007; Bargain and Donni, 2010; Lewbel and Pendakur, 2008; Lise and Seitz, 2007). When you think about reasons why this assumption might not hold (preferences for time might differ accross family status), two main reasons come in mind: heterogeneity and welfare interactions. Both reasons are controlled by the model so this assumption is not as strong as it might appear at first sight. First, in the empirical specification, preference heterogeneity is included so this assumption of preference equality is applied conditionnal on observed characteristics. Second, welfare interactions due to time-use 
in the family should be adequately controlled for. The time-use technology function does this. Our view is that it is the case that this function adequately summarizes these interactions. However, we recognize that this assumption could be problematic the marriage or divorce process in itself is related with time-use preferences or time-use interaction in the family, which is a situation that could not be formally excluded.

We know turn to the second condition. Structural estimation of the timeuse demand function for couples can only be complete if the identification of time-use demand is achieved for singles. This is problematic since prices remain fixed for singles. So generally, price elasticities of each activity cannot be individually identified. Indeed, if we denote $h$ the structural time-use demand for singles and $\zeta$ the reduced-form one, we have that :

$$
t^{k}=h^{k}\left(p^{1}, \ldots, p^{K}, \widetilde{F I}\right)=\zeta^{k}(w, \widetilde{F I})
$$

where $\widetilde{F I}$ is the full income minus consumption and $p^{1}=\ldots=p^{K}=w$. In this case, price effects cannot be separately identified since $\zeta$ is observed whereas $h$ is not:

$$
\frac{\partial \zeta_{k}}{\partial w}=\Sigma_{j=1}^{K} \frac{\partial h_{k}}{\partial p_{j}}
$$

Assumption 2: Preferences are strongly separable and additive, and income effects vary across activities.

This assumption leads to a direct utility function $U($.$) which is made up of$ sub-utility functions for each commodity group $\left(t^{k}\right)$ combined additively, up to any monotonic transformation $\mathrm{F}: U=F\left(u^{1}\left(t^{1}\right)+u^{2}\left(t^{2}\right)+\ldots+u^{K}\left(t^{K}\right)\right)$. The strong separability assumption for additive preferences allows to identify all price effects using only one price variation and wealth effects $!^{5}$ It is

\footnotetext{
${ }^{5}$ See Deaton (1980) p.137.
} 
a necessary assumption to identify price effects in the time-use case since prices are the same across various activities. Hence, all prices effects and are identified. Indeed, cross price substitution effects across time-uses are a function of income effects, up to a multiplicative constant.

A Stone-Geary utility function presents the interesting property that price derivatives can be identified using only variations on wage and full income data. This certainly eases identification in our context since we do not have any price variation of time-use activities. The only variation is income.

Following Browning et al. (2006) and Chiappori and Ekeland (2006) we obtain a generic identification of the model. For $K \geq 1$ generic identification of the time-use technology parameters of $\mathbf{A}$ and the individual opportunity costs $\lambda_{f}, \lambda_{m}$ is achieved. It is straightforward to show that we have more equations than unknowns. Let's consider an assignable time $j$ that is purely private and for which $\alpha^{j}=0$. Can we identify the $K-1$ parameters of the time-use technology function and the two individual opportunity costs $\lambda_{f}, \lambda_{m}$ from the observation of the reduced form time-use functions (Equation (7)) ? Hence, we have $K+1$ unknowns and $2 K$ independent equations. If $K \geq 1$, then $2 K \geq K+1$ and the number of equations exceeds the number of unknowns. Given identification of the $K-1$ parameters $\alpha$ and the two individual opportunity costs $\lambda_{f}, \lambda_{m}$, we have $\mathbf{A}, \boldsymbol{\pi}_{f}$ and $\boldsymbol{\pi}_{m}$. Then, we have identification of $F(\mathbf{z})=\mathbf{A}\left(\mathbf{t}_{f}, \mathbf{t}_{m}\right)$. The private equivalents are identified from Equation (1).

\subsection{Equivalent Income and Indifference Scale}

Economies of scale measure the extra time that two singles living apart need to be as well of as living together. The gains associated with household jointness of time-use can be unequally shared within the family. Economies 
of scale in time-use represents the cost in time needed to consume the time private equivalents $\left(\sum_{k=1}^{n}\left(z_{f}^{k}+z_{m}^{k}\right)\right)$ in comparison to what the household spends $(\tilde{T})$. Following BCL, we define the relative economies of scale in time-use, $e$, by:

$$
e=\frac{\mathbf{p}^{\prime}\left[\sum_{k=1}^{n}\left(z_{f}^{k}+z_{m}^{k}\right)-\tilde{T}\right]}{\mathbf{p}^{\prime} \tilde{T}}=\frac{\sum_{k=1}^{n}\left(z_{f}^{k}+z_{m}^{k}\right)}{\tilde{T}}-1
$$

where $\mathbf{p}$ is a vector of one.

Indifference scales $I S_{i}$ represent the fraction of household time resources that a single $i$ would require to consume the time private equivalents $\mathbf{z}_{i}$ at market prices that put her on the same indifference curve that she attained as living in a couple. Equivalence scales are defined as:

$$
I S_{i}=\frac{\operatorname{Min}_{\mathbf{z}_{i}^{*}}\left(\mathbf{p}^{\prime} \mathbf{z}_{i}^{*} \mid u_{i}\left(\mathbf{z}_{i}^{*}\right)=u_{i}\left(\mathbf{z}_{i}\right)\right)}{\mathbf{p}^{\prime} \tilde{T}}
$$

In expression (10), the numerator corresponds to the minimal time-expenditures spend by single individual to achieve the same welfare level as living in a couple; and the denominator corresponds to couple time-expenditure.

This concept involves the definition of the equivalent time resources $T_{i}^{*}$ describing the minimum amount of free-time called for consuming the vector of time private equivalent $\mathbf{z}_{i}$, which allows to attain the same welfare as in a couple. Equivalent income is defined as $T_{i}^{*}=\sum_{k=1}^{n}\left(z_{i}^{k}\right)$ and Individual indifference scale as the ratio $I S_{i}=\frac{T_{i}^{*}}{\tilde{T}}$. Individual $i$ receiving a fraction of household's time-resources $T_{i}^{*}$, could buy equivalent private time bundles on the market, that allow her to attain the same utility as a member of household, including time-use economies of scale. 


\section{Empirical Application}

\subsection{Data}

We use the UK Time Use Survey 2000 which measures the amount of time spent on various activities. Respondents are asked to complete two 24-Hour dairies (a working and a non-working day) broken down into tenminutes slots. We use the weighted mean of the two diaries. Hence, each amount of minutes presented here is the average daily time devoted to one activity. The questionnaire includes socio-demographic details and variables on employment and income.

Our sample contains 1,111 full-time workers between 16 and 65 years old. Thus, we exclude participation issues and analyze allocation of leisure time conditional to working hours ${ }^{6}$ For identification purposes, we sample single females (159), single males (194) and couples (379) with no one else present in the household. Table 1 presents descriptives statistics. A frequent problem in time use analysis is related to significant infrequency of reporting which could create artefact in results. Indeed, the zeros reporting for some activities could reflect infrequency or preferences (a 'corner solution'). Browning and Bonke (2006) propose a method to correct for infrequency bias assuming that some zeros are linked to preferences and others to infrequency. Concerning time use, it is difficult to make this kind of assumptions. We prefer to compute the average time devoted to each activities between the two diaries (working day and non-working day) to reduce the frequency of zeros and use aggregate definition of time.

\footnotetext{
${ }^{6}$ Available time is 24-Hours (1440 minutes) minus daily working hours, including training.
} 
Table 1: Descriptive Statistics

\begin{tabular}{|c|c|c|c|c|}
\hline Variable & Single female & Single male & Female in couple & Male in couple \\
\hline \multirow[t]{2}{*}{ Wage } & 7.07 & 6.19 & 5.02 & 6.90 \\
\hline & $(10.65)$ & $(3.90)$ & $(3.27)$ & $(9.74)$ \\
\hline \multirow[t]{2}{*}{ Age } & 40.27 & 39.31 & 41.20 & 43.25 \\
\hline & $(11.12)$ & $(10.93)$ & $(12.35)$ & $(12.45)$ \\
\hline \multirow[t]{2}{*}{ Education } & 3.77 & 3.61 & 3.36 & 3.41 \\
\hline & $(1.84)$ & (1.98) & (1.87) & $(1.93)$ \\
\hline \multirow[t]{2}{*}{ House ownership } & 0.75 & 0.64 & 0.84 & 0.84 \\
\hline & $(0.44)$ & $(0.48)$ & $(0.36)$ & $(0.36)$ \\
\hline \multirow[t]{2}{*}{ Car ownership } & 0.73 & 0.68 & 0.93 & 0.93 \\
\hline & $(0.45)$ & $(0.47)$ & $(0.26)$ & $(0.26)$ \\
\hline \multirow[t]{2}{*}{ Available Time ${ }^{a}$} & 1098.77 & 1054.57 & 1117.31 & 1060.26 \\
\hline & $(123.60)$ & $(148.32)$ & $(128.09)$ & $(135.10)$ \\
\hline \multirow{2}{*}{ Hours worked $^{a}$} & 320.49 & 371.72 & 309.44 & 368.99 \\
\hline & $(117.97)$ & $(138.84)$ & $(127.05)$ & $(134.59)$ \\
\hline \multirow[t]{2}{*}{ Sleep ${ }^{a}$} & 493.83 & 472.71 & 496.71 & 479.79 \\
\hline & $(73.73)$ & $(88.81)$ & $(68.41)$ & $(75.61)$ \\
\hline \multirow[t]{2}{*}{ Personal care ${ }^{a}$} & 124.47 & 109.55 & 126.66 & 117.00 \\
\hline & $(47.79)$ & $(52.41)$ & $(48.82)$ & $(53.39)$ \\
\hline \multirow[t]{2}{*}{ Household work $^{a}$} & 144.87 & 96.44 & 173.42 & 111.72 \\
\hline & $(87.06)$ & $(78.58)$ & $(98.63)$ & $(82.59)$ \\
\hline \multirow[t]{2}{*}{ Pur leisure $^{a}$} & 252.74 & 277.29 & 231.70 & 264.16 \\
\hline & $(95.10)$ & $(122.34)$ & $(89.17)$ & $(106.57)$ \\
\hline \multirow[t]{2}{*}{$\operatorname{commuting}^{a}$} & 82.87 & 96.46 & 87.73 & 86.51 \\
\hline & $(42.47)$ & $(60.84)$ & $(51.71)$ & $(52.22)$ \\
\hline Observation & 159 & 194 & 379 & 379 \\
\hline
\end{tabular}

$a$ in minutes per day. Standard deviation in parentheses.

\subsection{Empirical Implementation}

\subsubsection{Empirical Specification}

We adopt a Stone-Geary utility function allowing identification of all price effects. For singles, the conditional program is:

$$
\left\{\begin{array}{l}
\max _{t^{1}, \ldots, t^{K}} \prod_{j=1}^{K}\left(t^{j}-\gamma^{j}\right)^{\rho^{j}} \\
\text { st. } \Sigma_{j=1}^{K} t^{j}=\widetilde{T}_{i}
\end{array}\right.
$$

where $\rho^{j}$ are the Stone-Geary parameters which are individual and goodspecific, which represent the marginal budget shares; $\gamma^{j}$ is the incompressible levels of consumption of time-activity $t^{j}$. The sum of all the proportions of the goods consumed must equal $1\left(\sum_{j=0}^{K} \rho^{j}=1\right.$ and $\left.0<\rho^{j}<1\right)$. The Stone- 
Geary utility function gives rise to the linear expenditure system. Adding observed and unobserved heterogeneity, the conditional demands of a single individual $i$ for time-activity $k$ have the following shape:

$$
t_{i}^{k}=\gamma_{i}^{k}+\rho_{i}^{k}\left(\widetilde{T}_{i}-\Sigma_{j=1}^{K} \gamma_{i}^{j}\right)+\varepsilon_{i}^{s}
$$

Parameters $\gamma_{i}^{k}$ and $\rho_{i}^{k}$ are estimated using singles data. We parametrize $\rho_{i}^{k}$ in the following way, including heterogeneity in income effects:

$$
\rho_{i}^{k}=\frac{e^{\mathbf{x}_{i} \beta_{i}}}{1+e^{\mathbf{x}_{i} \beta_{i}}}
$$

Concerning couples, starting from the decentralized program, time-use demand equations can be written in terms of shadow prices and the sharing rule:

$$
\left\{\begin{aligned}
t_{f}^{k} & =\frac{1}{1-\left(\alpha^{k}\right)^{2}}\left[\gamma_{f}^{k}+\frac{\rho_{f}^{k}}{\pi_{f}^{k}}\left(\eta_{f}-\Sigma_{j=1}^{K} \gamma_{f}^{j} \pi_{f}^{j}\right)-\alpha^{k}\left(\gamma_{m}^{k}+\frac{\rho_{m}^{k}}{\pi_{m}^{k}}\left(\eta_{m}-\Sigma_{j=1}^{K} \gamma_{m}^{j} \pi_{m}^{j}\right)\right)\right]+\varepsilon_{f}^{c} \\
t_{m}^{k} & =\frac{1}{1-\left(\alpha^{k}\right)^{2}}\left[\gamma_{m}^{k}+\frac{\rho_{m}^{k}}{\pi_{m}^{k}}\left(\eta_{m}-\Sigma_{j=1}^{K} \gamma_{m}^{j} \pi_{m}^{j}\right)-\alpha^{k}\left(\gamma_{f}^{k}+\frac{\rho_{f}^{k}}{\pi_{f}^{k}}\left(\eta_{f}-\Sigma_{j=1}^{K} \gamma_{f}^{j} \pi_{f}^{j}\right)\right)\right]+\varepsilon_{m}^{c}
\end{aligned}\right.
$$

where $\eta_{i}=\widetilde{F I}_{i}(\mathbf{x}, s)$ and $s$ includes female to male ratios of wage and education as distribution factors. It is worth noticing that we cannot identify the sharing rule $\eta_{i}$ as the time-use demands is homogeneous of degree zero. We assume that $\eta_{i}$ corresponds to $\widetilde{F I}_{i}$. Implicitly, it implies that we know the Pareto weight. The following estimation would be conditional to a given Pareto weight $\mu$.

As we consider that gains from living together and the degree of publicness of time-activity could vary across household, we introduce heterogeneity in $\alpha^{k}$ which depends on demographics (female and male age, age squared, qualification):

$$
\alpha_{i}^{k}=\alpha_{i}^{k}\left(\mathbf{x}_{i}\right)
$$


Equation (16) describes the structural form of the Lindahl prices which depend on the opportunity cost of non-market time $\lambda_{f}$ and $\lambda_{m}$ which are unknowns. They corresponds to the derivative of the indirect utility function in respect to the spare time. Last, economies of scale, equivalent income and individual indifference scale are computed.

\subsubsection{Estimation Strategy}

The system is estimated by means of the Generalized Method of Moments (GMM) for singles and couples, assuming intra-group correlation, i.e. that the error terms are correlated across activities within households but uncorrelated across households.7 Personal care is considered as assignable and $\alpha^{1}=0.8$ Socio-demographic controls are household net non-labor market weekly income, age, age squared, education, a dummy variable for house ownership, for car availability and four regional dummies. In couple estimations, female and male sets of socio-demographics are including in spite of high correlation within couples, as we assume that time allocation of each household member depends on both sets of individual characteristics.

To deal with the potential endogeneity of available time conditional to endogenous working hours, we estimate simultaneously time-use for four activities (personal care, housework, leisure and commuting) and time resources using exclusion restrictions. A natural instrument for working hours and, therefore for time resources, is wage considered as an opportunity cost. However, (Browning and Meghir, 1991) note that the use of wage as instrument leads to difficulties. Beyond the selection problem, which refers to observabil-

\footnotetext{
${ }^{7} \mathrm{GMM}$ estimators are efficient, even when there is heteroskedasticity of unknown form (which is not the case with 3SLS). The Pagan and Hall test for the presence of heteroskedasticity confirms that GMM are called for.

${ }^{8}$ To allow for adding-up the first activities (sleeping) was dropped and (n-1) activities are estimated: personal care, household work 'pure' leisure and commuting.
} 
ity of wages only for workers, wage could be endogenous, due to measurement error, omitted variable or reverse causality. As the average hourly wage is computed as the quotient of weekly earnings by weekly working hours, any measurement error should introduce a spurious negative correlation between this instrument and the endogenous variable, namely labor supply (Mroz, 1987). Besides, household work and namely child care could be bought on the market, especially for individual with a higher leisure opportunity cost. Moreover, if the price of some activities like commuting is correlated with wages, elasticities estimation could be biased. Second, wages are weakly correlated with the amount of working hours, because the labor market is characterized by labor contracts often defined by wage-hours package in the medium run.

Given those problems we prefer to use an alternative set of instruments determining wages and working hours. The complete set of instruments are demographics, a dummy variable for managerial responsibilities, for shift work and the local unemployment rate. As good predictors for wage estimation, work characteristics explain the amount of working hours and therefore, time resources. Good instruments should be both relevant and valid: correlated with the endogenous regressors and orthogonal to the errors. To check the relevance and the validity of our instrumental strategy, several tests was performed. The first stage of instrumentation is observed, to (i) observe the correlation between instruments, the endogenous variables and the dependent ones for validity purposes, and (ii) confirm the endogeneity of the interest variable: the time resources. The Durbin-Wu-Hausman tests reject the exogeneity of time resources for each activities by gender. Second, instruments are correlated with the endogenous variable but uncorrelated with the de- 
pendent ones. The F-statistic tests reject their weakness. $9^{9}$ For single females, single males and couples we do not reject the over-identification test, meaning that the model is valid. Thus the Hansen-Sargan tests of over-identification do not reject the validity of our full set of instruments at the $5 \%$ level. For singles the $\chi^{2}(31)$ is 42.1 for female and 43.7 for males. For couples the $\chi^{2}(60)$ is 80.2. It is worth noticing that for singles, the over-identifying restriction tests implicitly correspond to homogeneity and symmetry restrictions tests.

Finally, we include two distribution factors in the full income estimations: the female to male ratio of wage and qualification.

\subsection{Separability Tests}

Finally, we provide a separability test using a more general functional semi-parametric shape for time-use demands $G$. We model $G$ using a flexible second order polynomial of $\widetilde{T}$, consumption $C$ and demographics $X$ (age, age squared, education, region and house ownership):

$G_{2}(\widetilde{T}, C, \mathbf{x}, \boldsymbol{\alpha})=\alpha_{0}+\alpha_{1} \widetilde{T}+\alpha_{2} C+\alpha_{3} \mathbf{x}+\alpha_{4} \widetilde{T}^{2}+\alpha_{5} C^{2}+\alpha_{6} \mathbf{x}^{2}+\alpha_{7} C * \widetilde{T}+\alpha_{8} C * \mathbf{x}$

A simple test of weak separability consists of testing whether the timedemands $t_{i}^{k}$ depend on the quantities of goods purchased $C_{i}$. With the polynomial shape of our functional form, all we have to do is to test whether the marginal effect of consumption on time-use is zero for all individuals $i\left(H_{0}\right)$. If the null hypothesis (the marginal effect of consumption on time-use demand is zero) is rejected, it would be a statistical evidence against our separability assumption. We use the total net household weakly income to proxy con-

\footnotetext{
${ }^{9}$ One rule of thumb is that an F-statistic below 10 is cause for weakness concern (Staiger and Stock,1997). Here, the F-statistic is equal to 16.6 for female and 18.46 for male, and confirms the relevance of our instrument sets.
} 
sumption. As the consumption $C$ is endogenous, we use instruments that explain mainly hours of work and mainly belong to the employment characteristics: non-labor income, wage, a dummy variable if individual works full-time and their interactions.

Table 2: Separability Tests

\begin{tabular}{lcccc}
\hline \hline & Single female & Single male & Female in couple & Male in couple \\
\hline Personal care & -0.06 & -0.09 & $-0.09^{* *}$ & -0.05 \\
& $(-0.2)$ & $(-0.56)$ & $(-2.39)$ & -1.21 \\
Housework & -0.54 & 0.22 & -0.05 & $0.13^{* *}$ \\
& $(-1.15)$ & $(0.95)$ & $(-0.73)$ & $(2.4)$ \\
Leisure & 0.92 & -0.4 & -0.07 & -0.01 \\
\multirow{2}{*}{ Commuting } & $(1.83)$ & $(-1.24)$ & $(-1.15)$ & $(-0.01)$ \\
& -0.005 & -0.09 & -0.01 & -0.02 \\
Marginal effect. t statistic in parentheses. ${ }^{* *}$ significant at the 5\% level. &
\end{tabular}

Table 2 presents the separability tests. The marginal effects of consumption expenditures on time-use demands for each time-use activity presented here are evaluated at sample means. Observing the estimates of the total net household weakly income on time-demands we see that some of them are significant: the impact of on personal care for female in couple and on housework for male in couple. We conclude that separability between consumption and daily time-allocation assumption is rejected for couples but not for singles. For couples, rejection of separability could be linked to non-separable utility function or non-separable time technology function 10 To take into account the rejection of separability we adopt a conditional approach without separability by adding the household consumption in covariates for the time-use technology function:

\footnotetext{
${ }^{10}$ Using our functional form (Stone-Geary utility function), we do not reject the separability assumption for singles.
} 


$$
\alpha_{i}^{k}=\alpha_{i}^{k}\left(\mathbf{x}_{i}, C\right)
$$

The interpretation is interesting since $C$, or a share of $C$ can be interpreted has buying market substitutes for time.

\subsection{Results}

The first paragraph deals with estimation of single females and single males to recover the preference parameters. Table 3 shows the $\boldsymbol{\rho}$ parameters of the Stone-Geary function. They are computed for the average non-labor income, age, age squared, education, house ownership, car availability and regional dummies. These coefficients also correspond to the marginal spare time share of each activity in the spare time. A significant proportion of free-time is devoted to leisure: $42 \%$ for female and $43 \%$ for male. Gender differences are noted concerning the time devoted to household chores: women spend $29 \%$ of their spare time in household tasks while men spend about $17 \%$.

Table 3: Preference Parameters

\begin{tabular}{lcc}
\hline \hline & Single Female & Single Male \\
\hline Personal Care & $0.079^{* *}$ & $0.085^{* * *}$ \\
& $(0.0323)$ & $(0.0255)$ \\
Housework & $0.285^{* * *}$ & $0.168^{* * *}$ \\
& $(0.0467)$ & $(0.0355)$ \\
Leisure & $0.424^{* * *}$ & $0.431^{* * *}$ \\
& $(0.0534)$ & $(0.0504)$ \\
Commuting & $0.02^{* *}$ & $0.068^{* *}$ \\
Sleeping & $(0.0092)$ & $(0.0304)$ \\
\hline
\end{tabular}

Note that $\overline{\overline{\text { due to adding-up restriction, time devoted to sleeping }}}$ is not estimated. Then the proportion of free-time devoted to sleeping is computed as follow: $1-\Sigma_{k=1}^{4} \rho k$. Absolute standard errors are in parentheses. *,**,*** significant at the 10,5 and $1 \%$ level. 
Table 4 shows the estimated parameters of the time-use technology function $\boldsymbol{\alpha}$. As we include heterogeneity in the time-use technology parameters, results are estimated for the average sample. Results suggest that all timeactivities have a public and a private component. Lindahl prices are also

Table 4: Time-use technology function estimation and Lindahl Prices

\begin{tabular}{cccc}
\hline \hline & \multicolumn{1}{c}{$\alpha$} & $\pi_{f}$ & $\pi_{m}$ \\
\cline { 2 - 4 } Housework & $0.08^{* *}$ & $5.08^{* * *}$ & $5.37^{* * *}$ \\
& $(0.039)$ & $(0.33)$ & $(0.77)$ \\
Leisure & $0.22^{* *}$ & $4.08^{* * *}$ & $4.61^{* * *}$ \\
& $(0.088)$ & $(0.733)$ & $(0.773)$ \\
\multirow{4}{*}{ Commuting } & $0.08^{* *}$ & $20.31^{* * *}$ & $27.92^{* * *}$ \\
& $(0.038)$ & $(5.088)$ & $(5.471)$ \\
\cline { 2 - 4 } Standard & &
\end{tabular}

estimated in their reduced form. Table 4 presents the $\pi$ estimates. They correspond to the price of private time equivalent within the household. Female Lindahl prices are lower than those of male for all activities. It suggests that on average women purchase private time equivalent cheaper than men do within the household ${ }^{11}$ On average Lindahl prices for both spouses are lower than individual wages except for commuting. Taking into account economies of scale linked to living in a couple suggests that one hour devoted to housework or leisure purchased within the household is cheaper than their relative opportunity cost. In other words, time spent outside the labor market cost less than what the individual would earn by supplying this time on the labor market.

The overall scale economy measure indicates that two singles living apart need $6.3 \%$ time more to achieve the same utility level as living in couple and maintaining the same preferences. A couple save $2 \mathrm{~h} 15$ a day by living

\footnotetext{
${ }^{11}$ This is only true for the average sample of socio-demographics variables. For some couples, the inverse is observed.
} 
Table 5: Economies and Indifference Scales

\begin{tabular}{lcc}
\hline \hline & For 2 singles & \\
\hline Economies of Scale (\% of household spare time) & 0.063 & $(0.074)$ \\
Economies of Scale (minutes) & 135.60 & $(158.202)$ \\
Female Indifference Scale (\% of household spare time) & 0.54 & $(0.049)$ \\
Male Indifference Scale (\% of household spare time) & 0.52 & $(0.055)$ \\
\hline \hline \multicolumn{2}{c}{ Standard deviation are in parentheses.Computed at the average mean of the } \\
sample.
\end{tabular}

Table 6: Economies of Scales by Activity and Household Member

\begin{tabular}{lcc}
\hline \hline & Female & Male \\
\hline Housework (\% of time devoted to) & 0.13 & 0.21 \\
minutes & 22.13 & 17.49 \\
Leisure (\% of time devoted to) & 0.31 & 0.22 \\
minutes & 71.82 & 58.71 \\
Commuting (\% of time devoted to) & 0.09 & 0.13 \\
minutes & 8.31 & 6.95 \\
\hline Computed at the average mean of the sample.
\end{tabular}

together. This estimation of economies of scale is an upper bound because differences between market and shadow prices suggest that singles can reallocate and more cheaply attain the same indifference curves: a woman (men) requires $55 \%$ (52\%) of joint time-resources to be as well when she (he) lives alone.

Then, economies of scale by activity and household member is presented in Table 6. Living in couple allows to save time. On average, a female wins 22 minutes of time devoted to housework, $72 \mathrm{~min}$ of leisure and 8 minutes of commuting per day. Living with his wife gives rise to economies of scale for married male: 20 minutes saved in housework, 60 minutes of leisure and 7 minutes of commuting to attain the same level of well-being. Obviously, the time saving depends on the characteristics of the couple and some wife could for example loose time by living with her partner. 
Last, poverty line are computed in three steps following Cherchye, De Reck and Vermeulen (2012). First, total expenditures on private time equivalent are computed. Based on our collective approach, it corresponds to the sum of the private equivalent commodities $z^{k}$. For singles, it corresponds to the sum of $t^{k}$, that is total time resources $\widetilde{T}$, as $z^{k}=t^{k}$ Obviously, these amounts depends on the level of economies of scales, for a given Pareto weight. Second, the median is computed. Third, individual with less than $60 \%$ of median equivalent time expenditures is considered to be time-poor. For the complete sample, the median equivalent time expenditures is about 18h30. Hence, the time-poverty line is around $11 \mathrm{~h}$ per individual a day ${ }^{13} \mathrm{It}$ represents $3 \%$ of our sample, that is full-time worker living alone or with his partner without children or anydbody else in the household.

\section{Conclusion}

This paper analyzes equivalence scales and economies of scales in time use. We compare individual time-welfare between two marital situations: single and couple. Following BCL, our equivalence scales overtake previous shortfall in the related literature.

Our contribution remains limited from policy perspectives since households with children are excluded and we focus on short-term choices. Still, this model brings a first stone in the building of a complete model of consumption and time-use intra-household welfare interaction. Further research directions would combine economies of scale in consumption and in time-use, fully release the separability assumption and generalize the time-use technology specification.

\footnotetext{
${ }^{12}$ It is worth noticing that there are valued at market prices $p=1$.

${ }^{13}$ Reminder that is an average for working and non-working day.
} 
An interesting and promising approach taken in this paper is to provide an individually-based definition of time-poverty. This could be used to define an individual compensation after a divorce or a partner's death, or to compute and compensate costs related to children. To be fully operationnal from a policy perspective, a more complete model that would allow describing the link between income and time poverty is necessary. We could then move from a purely material redistributive aim to a broadly redistributive aim including the assessment of spare time. 


\section{Bibliography}

[1] Apps, Patricia F., and Rees, Ray. 1988. Taxation and the household, 35(3), 355-369.

[2] Banks, James, Blundell, Richard, \& Lewbel, Arthur. 1997. Quadratic Engel Curves And Consumer Demand. The Review of Economics and Statistics, 79(4), 527-539.

[3] Bargain, Olivier, \& Donni, Olivier. 2012. The Measurement of Child Costs: A Rothbarth-Type Method Consistent with Scale Economies and Parents' Bargaining. forthcoming in the European Economic Review, Sept.

[4] Bloemen, Hans G. 2010. An Empirical Model of Collective Household Labour Supply with Non-Participation. Economic Journal, 120(543), $183-214$.

[5] Blundell, Richard, Chiappori, Pierre-André, \& Meghir, Costas. 2005. Collective Labor Supply with Children. Journal of Political Economy, 113(6), 1277-1306.

[6] Browning, Martin, \& Bonke, Jens. 2006. Allocation within the household: direct survey evidence. University of Oxford Working Paper.

[7] Browning, Martin, \& Chiappori, Pierre-André. 1998. Efficient IntraHousehold Allocations: A General Characterization and Empirical Tests. Econometrica, 66(6), 1241-1278.

[8] Browning, Martin, \& Meghir, Costas. 1991. The Effects of Male and Female Labor Supply on Commodity Demands. Econometrica, 59(4), $925-51$. 
[9] Browning, Martin, Bourguignon, Francois, Chiappori, Pierre-André, \& Lechene, Valerie. 1994. Income and Outcomes: A Structural Model of Intrahousehold Allocation. Journal of Political Economy, 102(6), 106796.

[10] Browning, Martin, Chiappori, Pierre-André, \& Lewbel, Arthur. 2006. Estimating Consumption Economies of Scale, Adult Equivalence Scales, and Household Bargaining Power. Oxford Economics Series Working Papers.

[11] Cherchye, Laurens, De Rock, Bram, \& Vermeulen, Frederic. 2012. Economic Well-Being and Poverty Among the Elderly: An Analysis Based on a Collective Consumption Model. European Economic Review, 56(6), 985-1000.

[12] Chiappori, Pierre-André, \& Donni, Olivier. 2006. Les modèles non unitaires de comportement du ménage : un survol de la littérature. L'Actualité Economique, 82(1), 9-52.

[13] Chiappori, Pierre-André, \& Ekeland, Ivar. 2006. The micro economics of group behavior: General characterization. Journal of Economic Theory, 130(1), 1-26.

[14] Couprie, Hélène. 2007. Time allocation within the Family: Welfare implications of life in a couple. Economic Journal, 117(01), 287-305.

[15] Deaton, Angus, \& Muellbauer, John. 1980. Economics and Consumer Behavior. Cambridge Books, no. 9780521296762. Cambridge University Press.

[16] Donni, Olivier. 2009. A Simple Approach to Investigate Intrahousehold 
Allocation of Private and Public Goods. The Review of Economics and Statistics, 91(3), 617-628.

[17] Fortin, Bernard, \& Lacroix, Guy. 1997. A Test of the Unitary and Collective Models of Household Labour Supply. Economic Journal, 107(443), 933-55.

[18] Goodin, Robert E, Rice, James Mahmud, Bittman, Michael, \& Saunders, Peter. 2005. The Time Pressure Illusion: Discretionary Time vs Free Time. Social Indicators Research, 73, 43-70.

[19] Gronau, Reuben. 1988. Consumption Technology and the Intrafamily Distribution of Resources:. Journal of Political Economy, 96(6), 11831205 .

[20] Hamermesh, Daniel S, \& Lee, Jungmin. 2007. Stressed Out on Four Continents: Time Crunch or Yuppie Kvetch? The Review of Economics and Statistics, 89(2), 374-383.

[21] Ipsos-RSL and Office for National Statistics, United Kingdom Time Use Survey. 2000 [computer file]. 3rd Edition. Colchester, Essex: UK Data Archive [distributor], September 2003. SN: 4504, http://dx.doi.org/10.5255/UKDA-SN-4504-1

[22] Lechene, Valerie. 1993. Une revue de la litterature sur les echelles d'equivalence. DELTA Working Papers.

[23] Lewbel, Arthur, \& Pendakur, Krishna. 2008. Estimation of collective household models with Engel curves. Journal of Econometrics, 147(2), $350-358$. 
[24] Lise, Jeremy, \& Seitz, Shannon. 2007. Consumption inequality and intra-household allocations. IFS Working Papers, July.

[25] Mroz, Thomas A. 1987. The Sensitivity of an Empirical Model of Married Women's Hours of Work to Economic and Statistical Assumptions. Econometrica, 55(4), 765-99.

[26] Nelson, Julie A. 1988. Household Economies of Scale in Consumption: Theory and Evidence. Econometrica, 56(6), 1301-14.

[27] Nelson, Julie A. 1993. Household Equivalence Scales: Theory versus Policy? Journal of Labor Economics, 11(3), 471-93.

[28] Pollak, Robert A, \& Wales, Terence J. 1979. Welfare Comparisons and Equivalence Scales. American Economic Review, 69(2), 216-21.

[29] Pollak, Robert A., \& Wales, Terence J. 1995. Demand System Specification and Estimation. OUP Catalogue, no. 9780195101218. Oxford University Press.

[30] Sofer, Catherine. 1999. EgalitÃl' entre femmes et hommes : aspects Âl'conomiques. Conseil d'Analyse Economique, La Documentation Française, Paris. Chap. Modélisation Economique de la prise de décision dans la famille.

[31] Staiger, Douglas, \& Stock, James H. 1997. Instrumental Variables Regression with Weak Instruments. Econometrica, 65(3), 557-586.

[32] Van Hoa, Tran, \& Ironmonger, Duncan Standon. 1989. Equivalence scales : A household production approach. Economics Letters, 31(4), $407-410$. 
[33] Vermeulen, Frederic. 2002. Collective Household Models: Principles and Main Results. Journal of Economic Surveys, 16(4), 533-64.

[34] Vermeulen, Frederic, \& Watteyne, Andrã C. 2006. Quand un et un ne font plus deux. L'Actualit Âl' Economique, 82(1), 155-180.

[35] Vicker, Clair. 1977. The Time-Poor: A New Look at Poverty. The Journal of Human Resources, Vol. 12, No. 1 (Winter, 1977), pp. 27-4, 12, $27-48$.

[36] Zeckhauser, Richard J. 1973. Time as the Ultimate. The Quarterly Journal of Economics, 87(4), 668-75. 


\section{Appendix}

\section{A. Individual Lindahl Prices}

We inverse equation (see Equation 1) with respect to individual time-uses $\mathbf{t}_{f}, \mathbf{t}_{m}$. This requires that the $\mathbf{A}$ matrix is not singular:

$$
\left\{\begin{array}{c}
t_{f}^{k}=\frac{z_{f}^{k}-\alpha^{k} z_{m}^{k}}{1-\left(\alpha^{k}\right)^{2}} \\
t_{m}^{k}=\frac{z_{m}^{k}-\alpha^{k} z_{f}^{k}}{1-\left(\alpha^{k}\right)^{2}}
\end{array}, \text { for } k=1, \ldots, K\right.
$$

Combining the time-use technology constraints and the two budget constraints leads to:

$$
\text { s.t. }\left\{\begin{array}{c}
1-\Sigma_{k=1}^{K}\left[\frac{z_{f}^{k}-\alpha^{k} z_{m}^{k}}{\widehat{T_{f}\left(1-\left(\alpha^{k}\right)^{2}\right)}}\right]=0 \\
1-\sum_{k=1}^{K}\left[\frac{z_{m}^{k}-\alpha^{k} z_{f}^{k}}{\widehat{T_{m}}\left(1-\left(\alpha^{k}\right)^{2}\right)}\right]=0
\end{array}\right.
$$

We apply the preceeding constraints to the centralized household program $\left(P^{c}\right)$ in order to compute necessary conditions. Then, we define following dual Program that leads to the same demands:

$$
\left(P^{d}\right)\left\{\begin{array}{l}
\max _{z_{f}, z_{m}} \mu u_{f}\left(z_{f}^{1}, \ldots, z_{f}^{K}\right)+u_{m}\left(z_{m}^{1}, \ldots, z_{m}^{K}\right) \\
\text { st. } \sum_{k=1}^{K} \frac{\pi_{f}^{k}}{\widetilde{F I_{f}}} z_{f}^{k}=1 \\
\sum_{k=1}^{K} \frac{\pi_{m}^{k}}{\overline{F I_{m}}} z_{m}^{k}=1
\end{array}\right.
$$

Equalizing cecessary conditions of the centralized program $\left(P^{c}\right)$ and the dual program $\left(P^{d}\right)$ leads to:

$$
\left\{\begin{aligned}
\pi_{f}^{k} & =\frac{w_{f}\left(\widetilde{T}_{m} \lambda_{f}-\widetilde{T}_{f} \lambda_{m} \alpha^{k}\right)}{\widetilde{T}_{m} \lambda_{f}\left[1-\left(\alpha^{k}\right)^{2}\right]} \\
\pi_{m}^{k} & =\frac{w_{m}\left(\widetilde{T}_{f} \lambda_{m}-\widetilde{T}_{m} \lambda_{f} \alpha^{k}\right)}{\widetilde{T}_{f} \lambda_{m}\left[1-\left(\alpha^{k}\right)^{2}\right]}
\end{aligned}\right.
$$




\section{B. Estimation Results}

Table 7: Single Estimations

\begin{tabular}{|c|c|c|c|c|c|c|c|c|c|c|}
\hline \multirow[b]{3}{*}{ Constant } & \multicolumn{10}{|c|}{ Single female } \\
\hline & \multicolumn{2}{|c|}{ Personal Care } & \multicolumn{2}{|c|}{ Housework } & \multicolumn{2}{|c|}{ Leisure } & \multicolumn{2}{|c|}{ Commuting } & \multicolumn{2}{|c|}{ Spare Time } \\
\hline & 0.082 & $(0.037)$ & 0.200 & $(0.062)$ & 0.488 & $(0.059)$ & 0.010 & $(0.0345$ & 1102.912 & $(12.353)$ \\
\hline Age & 0.000 & $(0.001)$ & 0.002 & $(0.002)$ & -0.002 & $(0.002)$ & 0.000 & $(0.001)$ & & \\
\hline Age squared & 0.000 & $(0.000)$ & 0.000 & $(0.000)$ & 0.000 & $(0.000)$ & 0.000 & $(0.000)$ & & \\
\hline Education & 0.001 & $(0.001)$ & -0.001 & $(0.001)$ & 0.000 & $(0.001)$ & 0.001 & $(0.000)$ & & \\
\hline Region & 0.006 & $(0.004)$ & 0.001 & $(0.007)$ & -0.016 & $(0.008)$ & 0.005 & $(0.004)$ & & \\
\hline House Ownership & 0.002 & $(0.004)$ & -0.001 & $(0.005)$ & 0.003 & $(0.006)$ & 0.001 & $(0.003)$ & & \\
\hline $\begin{array}{l}\text { Non-labor income } \\
\text { Manage }\end{array}$ & & & & & & & & & $\begin{array}{c}0.555 \\
-35.936\end{array}$ & $\begin{array}{c}(0.182) \\
(15.766)\end{array}$ \\
\hline $\begin{array}{l}\text { R-squared } \\
\text { Observation }\end{array}$ & & & & & & $\begin{array}{l}37 \\
156 \\
\end{array}$ & & 33 & & \\
\hline Observation & \multicolumn{10}{|c|}{ Single male } \\
\hline Constant & 0.077 & $(0.035)$ & 0.134 & $(0.049)$ & 0.414 & $(0.0703)$ & 0.042 & $(0.043)$ & 1046.932 & $(13.556)$ \\
\hline Age & -0.001 & $(0.001)$ & 0.001 & $(0.001)$ & 0.002 & $(0.003)$ & 0.001 & $(0.001)$ & & \\
\hline Age squared & 0.000 & $(0.000)$ & 0.000 & $(0.000)$ & 0.000 & $(0.000)$ & 0.000 & $(0.000)$ & & \\
\hline Education & 0.002 & $(0.001)$ & -0.001 & $(0.001)$ & -0.002 & $(0.002)$ & 0.001 & $(0.001)$ & & \\
\hline Region & 0.007 & $(0.067)$ & -0.019 & $(0.008)$ & 0.014 & $(0.011)$ & 0.005 & $(0.006)$ & & \\
\hline House Ownership & 0.000 & $(0.004)$ & 0.010 & $(0.006)$ & -0.014 & $(0.009)$ & 0.010 & $(0.005)$ & & \\
\hline Non-labor income & & & & & & & & & 0.970 & $(0.243)$ \\
\hline Manage & & & & & & & & & -15.329 & $(5.818)$ \\
\hline R-squared & \multirow{2}{*}{\multicolumn{2}{|c|}{0.33}} & \multirow{2}{*}{\multicolumn{2}{|c|}{0.3}} & \multirow{2}{*}{\multicolumn{2}{|c|}{$\begin{array}{c}0.36 \\
193\end{array}$}} & \multirow{2}{*}{\multicolumn{2}{|c|}{0.33}} & \multirow{2}{*}{\multicolumn{2}{|c|}{0.34}} \\
\hline Observation & & & & & & & & & & \\
\hline
\end{tabular}

Note that due to adding-up restriction, time devoted to sleeping is not estimated, Absolute standard errors are in parentheses. 
Table 8: Couple Estimations

\begin{tabular}{|c|c|c|c|c|c|c|}
\hline & $\alpha^{2}$ & & & $\alpha^{3}$ & $\alpha^{4}$ & \\
\hline Constant & -86.84 & $(36.321)$ & 6.09 & $(2.647)$ & -327.78 & $(142.111)$ \\
\hline Female Age & 0.45 & $(0.194)$ & 0.05 & $(0.0214)$ & 28.94 & $(11.408)$ \\
\hline Female Age squared & 0.00 & $(0.001)$ & 0.00 & $(0.000)$ & -0.63 & $(0.242)$ \\
\hline Female Education & 5.52 & $(2.33)$ & -0.47 & $(0.254)$ & 1.24 & $(0.538)$ \\
\hline Male Age & -0.10 & $(0.039)$ & 0.00 & $(0.001)$ & -0.03 & $(0.012)$ \\
\hline Male Age squared & 2.11 & $(0.961)$ & 0.08 & $(0.036)$ & -0.27 & $(0.116)$ \\
\hline Male Education & 0.24 & $(0.103)$ & -0.16 & $(-0.068)$ & -1.48 & $(0.352)$ \\
\hline \multirow[t]{2}{*}{ Consumption } & -0.04 & $(0.011)$ & 0.00 & $(0.001)$ & -0.02 & $(0.009)$ \\
\hline & & & Female Full Income & & Male Full Income & \\
\hline Constant & & & 53.42 & $(11.545)$ & 163.23 & $(29.237)$ \\
\hline Education ratio & & & 17.96 & $(3.914)$ & 1.77 & $(0.769)$ \\
\hline Wage ratio & & & 0.41 & $(0.027)$ & -1.57 & $(0.096)$ \\
\hline Partner's Spare time & & & 0.02 & $(0.010)$ & 0.11 & $(0.054)$ \\
\hline \multirow[t]{2}{*}{ Non-labor income } & & & 0.04 & $(0.010)$ & -0.03 & $(0.011)$ \\
\hline & $\pi_{f}^{2}$ & & $\pi_{f}^{3}$ & & $\pi_{f}^{4}$ & \\
\hline Constant & -0.13 & $(0.040)$ & -0.04 & $(0.017)$ & 2.47 & $(0.840)$ \\
\hline Female Age & 0.00 & $(0.001)$ & 0.00 & $(0.001)$ & -0.16 & $(0.045)$ \\
\hline Female Age squared & 0.00 & $(0.001)$ & 0.00 & $(0.001)$ & 0.00 & $(0.001)$ \\
\hline Male Age & 0.01 & $(0.002)$ & 0.00 & $(0.001)$ & 0.04 & $(0.008)$ \\
\hline Male Age squared & 0.00 & $(0.001)$ & 0.00 & $(0.001)$ & 0.00 & $(0.001)$ \\
\hline Education ratio & 0.03 & $(0.005)$ & 0.02 & $(0.005)$ & 0.04 & $(0.018)$ \\
\hline \multirow[t]{2}{*}{ Wage ratio } & 0.00 & $(0.001)$ & 0.00 & $(0.001)$ & 0.00 & $(0.001)$ \\
\hline & $\pi_{m}^{2}$ & & $\pi_{m}^{3}$ & & $\pi_{m}^{4}$ & \\
\hline Constant & 10.25 & $(1.460)$ & 5.50 & $(0.928)$ & 111.00 & $(17.752)$ \\
\hline Female Age & -0.56 & $(0.079)$ & -0.39 & $(0.056)$ & -8.89 & $(1.420)$ \\
\hline Female Age squared & 0.01 & $(0.001)$ & 0.01 & $(0.001)$ & 0.17 & $(0.026)$ \\
\hline Male Age & 0.002 & $(0.001)$ & 0.05 & $(0.018)$ & 0.94 & $(0.154)$ \\
\hline Male Age squared & -0.004 & $(0.001)$ & -0.01 & $(0.001)$ & -0.01 & $(0.002)$ \\
\hline Education ratio & 0.40 & $(0.055)$ & 0.28 & $(0.037)$ & 2.89 & $(0.456)$ \\
\hline Wage ratio & -0.89 & $(0.126)$ & -0.45 & $(0.045)$ & -6.78 & $(1.163)$ \\
\hline
\end{tabular}

